\title{
Diffuse Alveolar Hemorrhage as a complication of Churg Strauss
}

\section{Lovitt C., Mermis J.}

\section{Background}

Churg Strauss (CSS) is a multi-system disorder characterized by allergic rhinitis, asthma, and prominent peripheral blood eosinophilia. This disease is also classified as a vasculitis of small and medium sized arteries. CSS represents approximately $10 \%$ of diagnosed vasculitides. This disease is known to have multiple system effects including pulmonary infiltrates, mononeuritis multiplex, pericarditis, Gl

manifestations and skin manifestations.

\section{Case Report}

A 31 yo female presented to pulmonary clinic after being referred for possible cystic fibrosis. She had being referred for possible cystic fibrosis. She had been diagnosed with asthma in the past and had multiple pulmonary complaints with frequent exacerbations, as well as gastrointestinal complaints. She had frequent courses of steroids for asthma exacerbations. The work up for CF was negative but further work up revealed she had Churg Strauss. Following this diagnosis she began having pulmonary symptoms including cough, shorthess of breath and symptoms including cough, shotness of breath and hemoptysis. She was inithally treated with high dose steroids as well as broad spectrum antibiotics and began to improve. However, she again decompensated and required intubation. When the ET tube was placed, pulmonary suction revealed frank blood, her hematocrit had dropped from 37 to 27, and chest X-ray showed dense bilateral alveolar opacities. Subsequent CT showed scattered bilatera opacities. Subsequent CT showed scattered bilater pulmonary infiltrates and mild peribronchia thickening and were consistent with Diffuse alveolar Hemorrhage (DAH). Her treatment was escalated to cyclophosphamide along with steroids. Over the course of 4 weeks and treatment with

cyclophosphamide, this young patient slowly

recovered and was eventually discharged from the hospital.

\section{December - February}

\section{- \\ Patient presented to clinic with \\ uncontrolled asthma, she was on oral steroids and had recently been started on \\ Labs December WBC 5.6 with $15 \%$ eosinophils $\mathrm{Hgb} / \mathrm{Hct}: 13 / 39$ \\ Labs Early February P-ANCA: 160 \\ Myeloperoxidase AB: 7.6}

PFTs December

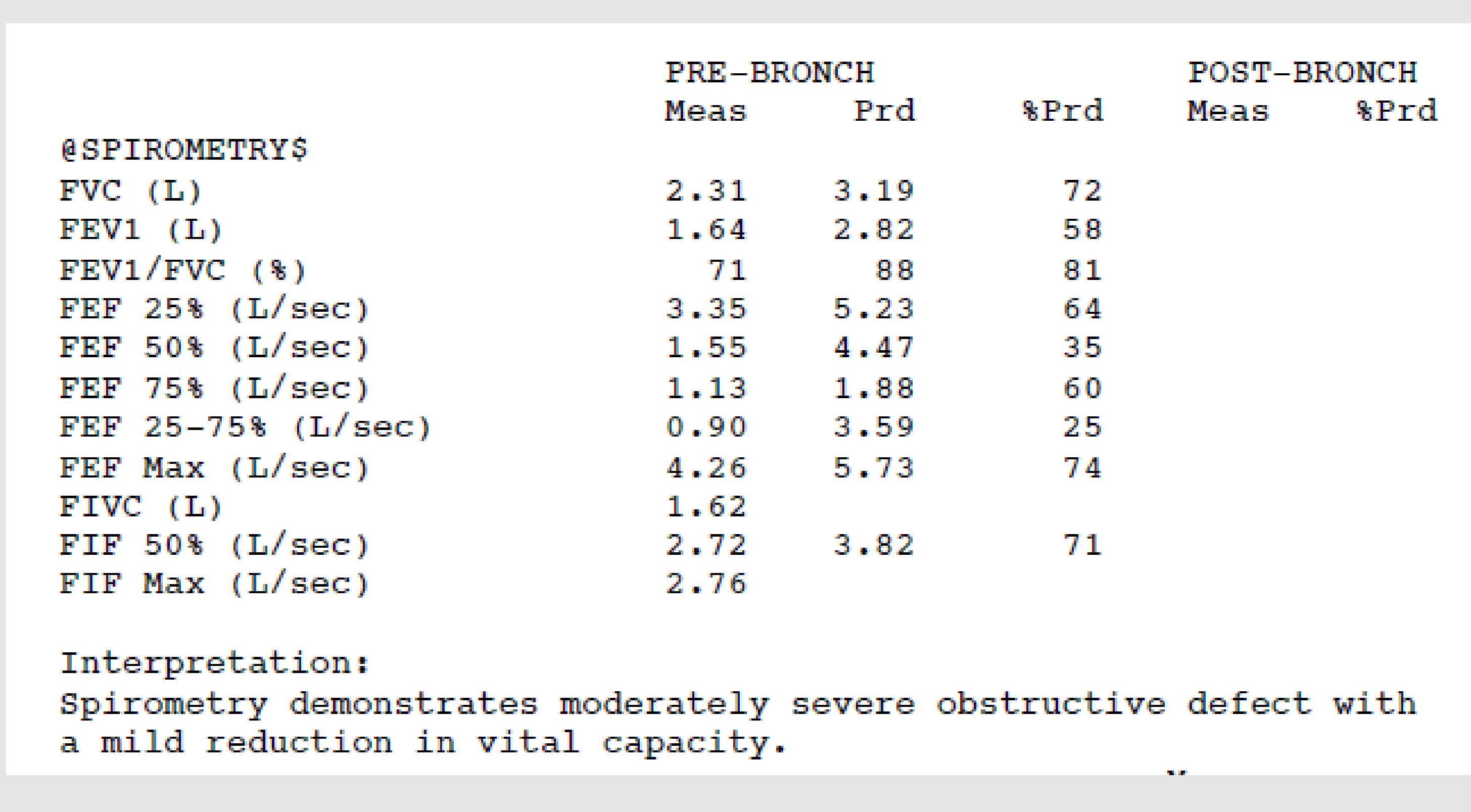

Chest $\mathrm{X}$ ray February 18 th

She then pres
hemoptysis.

Labs February 18th

$\mathrm{Hgb} / \mathrm{Hct:}: 13.9 / 40$
$\mathrm{ABG} 7.37 / 34 / 61$

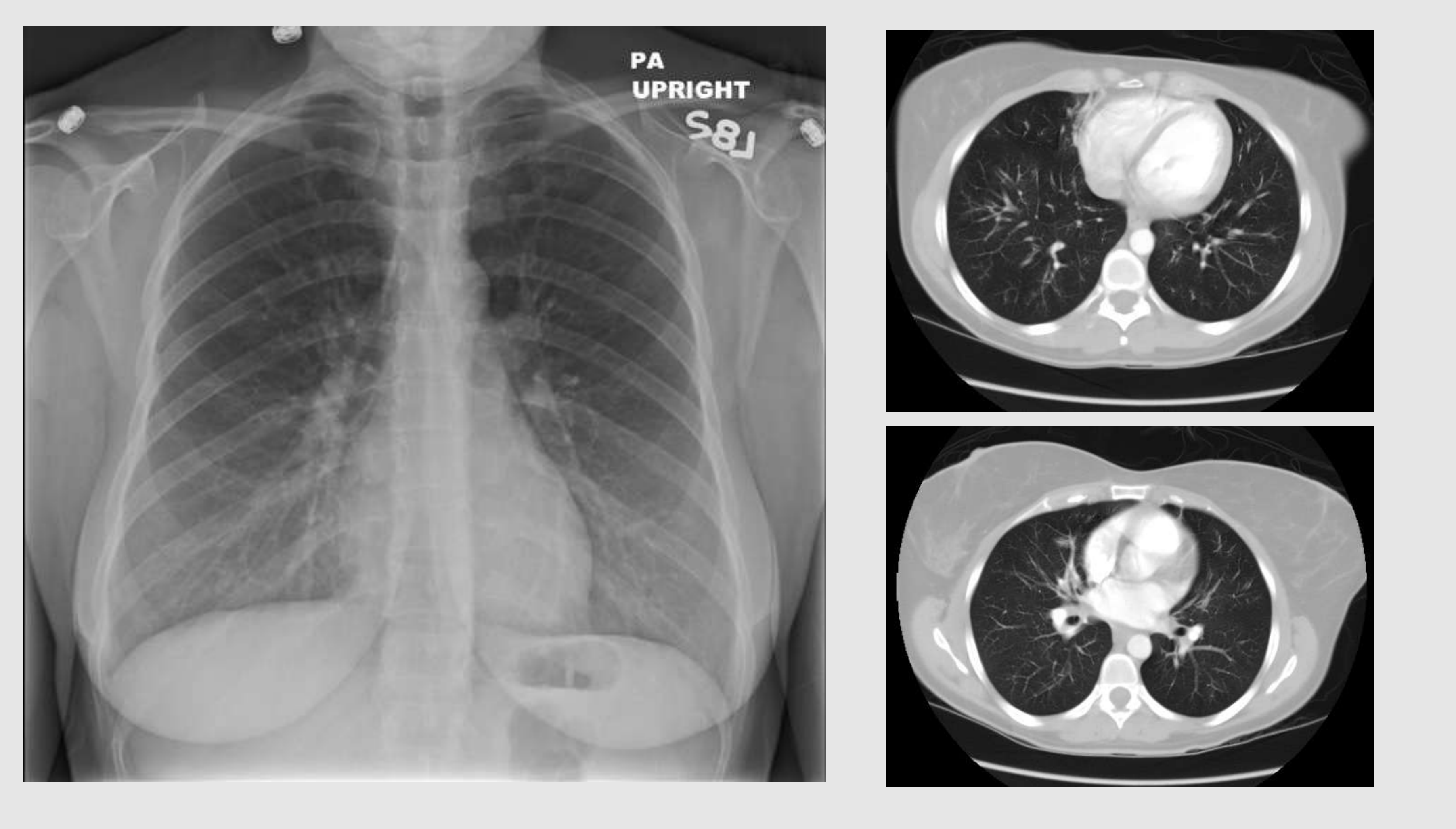

Within four days she rapidly declined with respiratory distress requiring intubation.

Labs February 22nd Hgb/Hct: 10/27
Chest $\mathrm{X}$ ray February 22nd

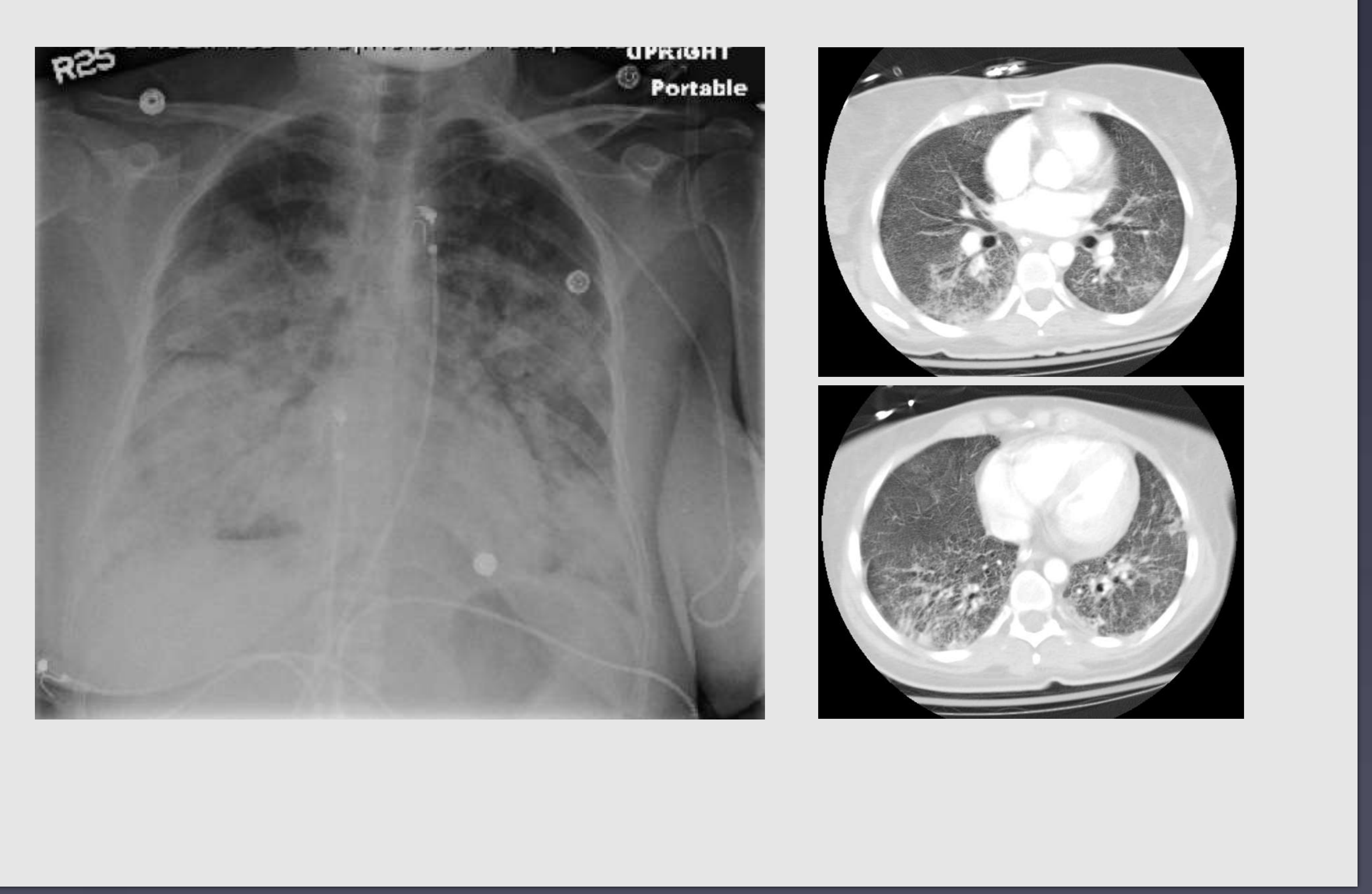

\section{Discussion}

DAH is characterized by hemoptysis (though this is not infrequently absent), fevers, chest pain,

cough, dyspnea and hypoxic respiratory failure. Laboratory examination shows falling hematocrit. process and is more detailed as to the extent of the disease process. For the diagnosis of this disease, flexible bronchoscopy should be done and samples. DAH is known to be associated with IPH, ANCA-Associated Granulomatous Vasculitis, MPA, SLE, mixed connective tissue disorders, and Goodpasture Syndrome, per Schwarz and Lara in May 2010. A case report from 1998 examined a patient with CSS who presented with respiratory symptoms and transbronchial biopsy revealed necrotizing vasculitis. On autopsy they discovered this patient had DAH, and their conclusion emphasized that CSS should be considered in patients who are found to have ANCA positive vasculitis and DAH. This case also supports that a patient with known CSS is at risk for DAH, and the early recognition of this will aid in rapid diagnosis and treatment of this frequently fatal complication. Imaging reveals a patchy, focal or diffuse alveolar filling process, and CT confirms this alveolar filling will show progressively hemorrhagic BAL in serial

\section{References}

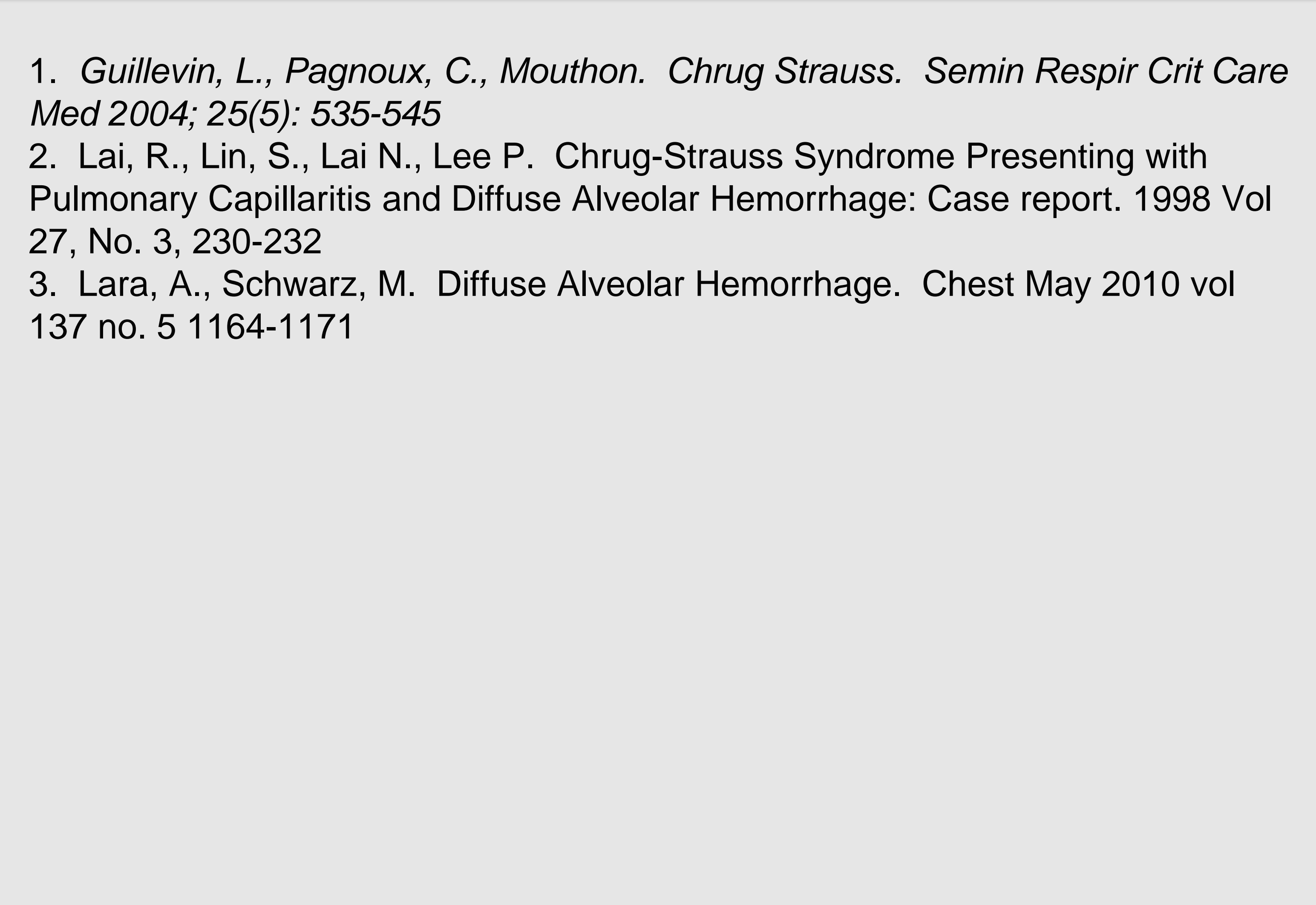

